\title{
Development of a Multi-Device Nutrition Logging Prototype Including a Smartscale
}

\author{
Andreas Seiderer \\ Augsburg University \\ Augsburg, Germany \\ seiderer@hcm-lab.de
}

\author{
Elisabeth André \\ Augsburg University \\ Augsburg, Germany \\ andre@hcm-lab.de
}

\begin{abstract}
In this work we present a portable system for nutrition logging that integrates multiple devices and modalities in order to facilitate food and drink tracking. The system consists of a smartphone, a smartwatch and a smartscale that can be combined flexibly by users depending on the current situation and their personal needs. Based on a requirement analysis, we present the rationale behind the design and implementation of our food and drink logger. We also report the preliminary results of an in-situ study we conducted in order to explore the potential benefits and challenges of a multidevice approach to nutrition tracking in daily life settings.
\end{abstract}

\section{CCS CONCEPTS}

-Human-centered computing $\rightarrow$ Mobile devices; User interface programming;

\section{KEYWORDS}

Nutrition Logging; Food Journal; Multi-Device; Smartwatch; In-Situ

ACM Reference format:

Andreas Seiderer and Elisabeth André. 2017. Development of a Multi-Device Nutrition Logging Prototype Including a Smartscale. In Proceedings of DH'17, fuly 2-5, 2017, London, United Kingdom, , 2 pages.

DOI: http://dx.doi.org/10.1145/3079452.3079486

\section{INTRODUCTION}

The type and amount of food and drink directly influences the health and overall well-being of a person. Thus, the logging of nutrition intake makes it possible to observe the effects on health and to detect improper nutrition. This can be especially helpful for people already suffering from diseases such as diabetes, overweight and food allergies, but also for others that wish to detect nutrient deficiencies or a too high calorie intake prematurely.

Nevertheless, recording the exact type and amount of each food and drink is a time-consuming task and often makes people stop it soon as they find the effort being too high [1]. Therefore, we present a prototype of a multi-device system consisting of a smartphone, a smartwatch and a smartscale and show how to leverage the situation-specific capabilities of the individual devices in order to reduce the effort. A smartphone provides flexible user input

(C) Author | ACM 2017. This is the author's version of the work. It is posted here for your personal use. Not for redistribution. The definitive Version of Record can be found at DOI: http://dx.doi.org/10.1145/3079452.3079486 possibilities and already significantly reduces the effort in comparison to traditional pen and paper methods [3]. A smartwatch can supplement a smartphone since it is always in reach and can be used in a less conspicuous manner. On the other hand, due to the small screen size, it is limited in its input capabilities. Often it is necessary to input the amount of foodstuff as accurate as possible which is usually a hard task. We therefore include a Bluetooth scale prototype (smartscale) in the system.

To be able to ensure user-friendly multi-device interaction we consulted the works by Dong et al. [2] and Sørensen et al. [5]. Dong et al. identified two dimensions of inter-device usability: knowledge continuity and task continuity that may be supported by a consistent user interface design to enable the user to easily switch from one device to the other. Sørensen et al. [5] distinguished between the simultaneous and sequential execution of tasks distributed over multiple devices which both occur during nutrition logging.

\section{REQUIREMENTS}

As a first step the requirements for our system were gathered by semi-structured interviews and by analyzing popular Android apps and related work. They include reducing logging effort, enhancing privacy and flexibility of use, generating reminders to log and offering possibilities to reflect about nutrition intake.

Multi-device interaction plays an important role in our system and requires a high amount of GUI consistency across all devices while following standards of the individual platforms [2]. Additionally, data synchronization between all devices [5] is required.

The requirements of our prototype are in line with the work by Lessel et al. [4]. Additionally, most importantly we wanted to avoid the effort of Bluetooth pairing and frequent battery charging and required a possibility to weigh plates larger than the top plate (not directly possible with a surrounding case rim).

\section{SYSTEM DEVELOPMENT}

The two smartscale prototypes, a modified kitchen scale and a custom construction (see Figure 1) use load cells as sensors as they deliver very clean data. With its integrated via USB adapter chargeable 3.4 Ah lithium battery it can run for several weeks. Bluetooth advertisement packets with the current weight as additional payload are used to save power and to omit Bluetooth pairing.

Our software prototypes on the smartphone and smartwatch use the Android platform. During the development process we first developed a low fidelity prototype that was evaluated in a heuristic evaluation to eliminate most severe usability problems.

Both prototypes use the same screen order and a similar GUI layout as far as it is possible as the smartwatch is limited in its input possibilities. Our smartphone prototype additionally allows 

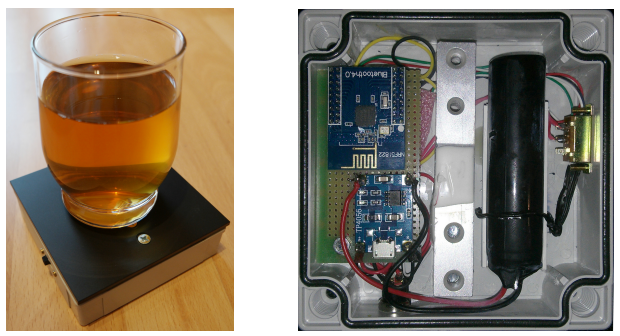

Figure 1: Smartscale prototype: compact construction (10.0 $x 10.0 \times 3.2 \mathrm{~cm}$ ) using the bottom half of an ABS plastic case and an acrylic glass top plate

inputting data via speech recognition (single text fields) and text input. The user can also edit the timestamp and add a photo and notes to an entry. On both devices the foodstuff can also be rated. The smartphone contains a database with foodstuff (around 30.000 German entries gathered from multiple sites on the Internet) that is used for auto completion. On the smartwatch it is reduced to around (7.000 entries). On the smartphone a widget and on the smartwatch a custom watch face are used to allow quick access to the apps. If the input data for the smartwatch are incomplete (e.g missing weight) these entries are highlighted on the smartphone overview and can be completed later on. From the overview also other entries can be edited and supplemented with additional data such as a photo or notes.

The data is being synchronized between the devices as shown in Figure 2. All components of the system are visible in Figure 3.

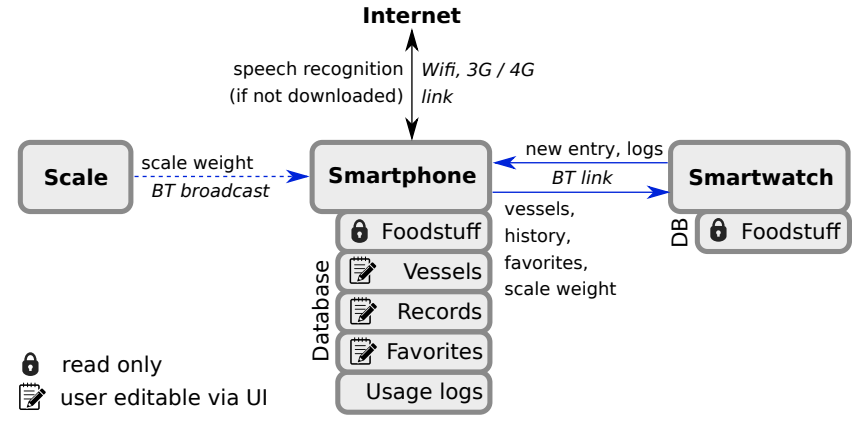

Figure 2: Communication between the scale, smartphone, smartwatch and the Internet

As the main task of the system is easy nutrition logging we optimize it by allowing the optional use of the smartscale for weight, optional subtraction of a vessel weight (editable list), auto completion / selection from a list, favorites and recently used entries.

\section{EVALUATION AND PRELIMINARY RESULTS}

We conducted a preliminary in-situ study with the prototype where we gave the system for one day to five people (24-31 years old) that were interested in logging their nutrition. After the usage of the prototype the study participants were interviewed.

The scale was considered as "an absolute must" to record the amount of food with a sufficient degree of accuracy. Nevertheless, it

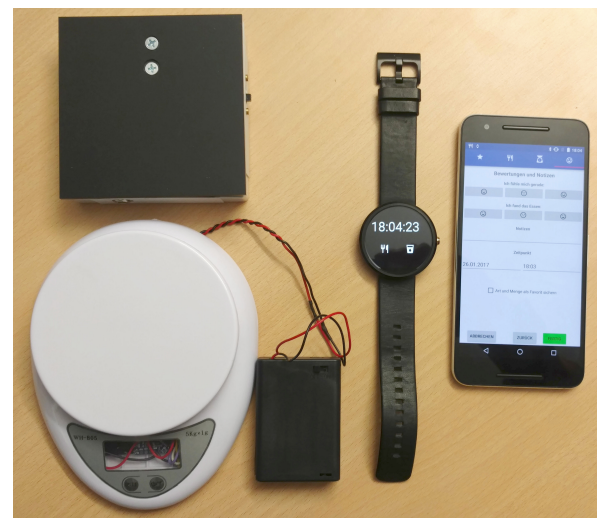

Figure 3: All devices used for the high fidelity system prototype: the two Bluetooth scales, a Moto 360 smartwatch and a Nexus 6P smartphone)

was just used at home. While users relied on the smartwatch when repeated entries had to be made, they preferred the smartphone for inputting new food categories or for providing more details on data that was previously recorded with the watch.

\section{CONCLUSION}

Our presented nutrition logging system offers greater flexibility to users than currently available smartscale systems that typically rely on a smartphone only as an interaction device. A preliminary insitu study revealed that users indeed made use of all three devices and exploited the advantages of all of them in a situation-specific manner.

We are currently planning to conduct a longer study with more participants and working on system improvements for example by adding smart reminders.

\section{REFERENCES}

[1] F. Cordeiro, D. A. Epstein, E. Thomaz, E. Bales, A. K. Jagannathan, G. D. Abowd, and J. Fogarty. 2015. Barriers and Negative Nudges: Exploring Challenges in Food Journaling. In Proceedings of the 33rd Annual ACM Conference on Human Factors in Computing Systems (CHI '15). ACM, New York, NY, USA, 1159-1162. DOI : http://dx.doi.org/10.1145/2702123.2702155

[2] T. Dong, E. F. Churchill, and J. Nichols. 2016. Understanding the Challenges of Designing and Developing Multi-Device Experiences. In Proceedings of the 2016 ACM Conference on Designing Interactive Systems (DIS '16). ACM, New York, NY, USA, 62-72. DOI : http://dx.doi.org/10.1145/2901790.2901851

[3] Y. Kim, S. Ji, H. Lee, J. Kim, S. Yoo, and J. Lee. 2016. "My Doctor is Keeping an Eye on Me!": Exploring the Clinical Applicability of a Mobile Food Logger. In Proceedings of the 2016 CHI Conference on Human Factors in Computing Systems (CHI '16). ACM, New York, NY, USA, 5620-5631. DOI : http://dx.doi.org/10.1145/ 2858036.2858145

[4] P. Lessel, M. Altmeyer, F. Kerber, M. Barz, C. Leidinger, and A. Krüger. 2016. WaterCoaster: A Device to Encourage People in a Playful Fashion to Reach Their Daily Water Intake Level. In Proceedings of the 2016 CHI Conference Extended Abstracts on Human Factors in Computing Systems (CHI EA '16). ACM, New York, NY, USA, 1813-1820. DOI : http://dx.doi.org/10.1145/2851581.2892498

[5] H. Sørensen, D. Raptis, J. Kjeldskov, and M. B. Skov. 2014. The 4C Framework: Principles of Interaction in Digital Ecosystems. In Proceedings of the 2014 ACM International foint Conference on Pervasive and Ubiquitous Computing (UbiComp '14). ACM, New York, NY, USA, 87-97. DOI : http://dx.doi.org/10.1145/2632048. 2636089

\section{COMPETING INTERESTS}

The authors have declared that no competing interests exist. 